\title{
Charge and Statistics of Quasiparticles in Fractional Quantum Hall Effect
}

\author{
B. Basu P. Bandyopadhyay甘 and S. Dhan \\ Physics and Applied Mathematics Unit \\ Indian Statistical Institute \\ Kolkata-700108
}

\begin{abstract}
We have studied here the charge and statistics of quasiparticle excitations in FQH states on the basis of the Berry phase approach incorporating the fact that even number of flux quanta can be gauged away when the Berry phase is removed to the dynamical phase. It is observed that the charge $q$ and statistical parameter $\theta$ of a quasiparticle at filling factor $\nu=\frac{n}{2 p n+1}$ are given by $q=\left(\frac{n}{2 p n+1}\right) e$ and $\theta=\frac{n}{2 p n+1}$, with the fact that the charge of the quasihole is opposite to that of the quasielectron. Using Laughlin wave function for quasiparticles, numerical studies have been done following the work of Kjønsberg and Myrheim [1] for FQH states at $\nu=1 / 3$ and it is pointed out that as in case of quasiholes, the statistics parameter can be well defined for quasielectrons having the value $\theta=1 / 3$.

PACS numbers:
\end{abstract}

\section{INTRODUCTION}

In an earlier work Kjønsberg and Myrheim [1] have reported that when one uses Laughlin wave function for fractional quantum Hall $(\mathrm{FQH})$ states, the corresponding quasielectron (QE) excitations are found to have charge and statistics different from those of quasiholes $(\mathrm{QH})$. In fact their numerical studies show that for system sizes upto 200 electrons, quasiholes have the charge and statistics parameter $e / 3$ and $1 / 3$ respectively. However for quasielectrons $(\mathrm{QE})$ the trends regarding the charge show a slow convergence towards the expected value $-e / 3$ and with a finite size correction for $N$ electrons approximately $-0.13 e / N$, the statistics parameter $\theta$ has no defined value but might probably converge to $1 / 3$ in the thermodynamic limit. On the other hand Kjønsberg and Leinaas 2] have shown that the composite fermion (CF) picture leads to a definite statistics parameter for a CFQP. Jeon et.al [3, 4] have concluded that the composite fermion $(\mathrm{CF})$ picture gives rise to the CFQP charge and statistics parameter given by $e^{*}=-\frac{e}{2 p n+1}$ and $\theta=\frac{2 p}{2 p n+1}$ for the filling factor $\nu=\frac{n}{2 p n+1}$. However numerical studies [3, 4] using CF wave function show that at filling factor $\nu=1 / 3$ and $2 / 5$ the statistics parameter $\theta$ is found to be $-2 / 3$ and $-2 / 5$ respectively. Though the numerical values agree with the theoretical predictions on the basis of $\mathrm{CF}$ model, it has opposite sign. The sign discrepancy would cast doubt on the fundamental interpretation of the CF physics. To cope with this problem these authors have suggested that the insertion of one composite fermion quasiparticle (CFQP) at a certain position is slightly perturbed by inserting another CFQP inside the loop which pushes the other very slightly outward.

From the above findings it appears that any of the models of quantum Hall fluid seem to satisfy the observations in an unambiguous way. Here we shall try to show that Laughlin wave function appears to lead to the charge and statistics parameter for QE compatible with $\mathrm{QH}$ when we take into account that the Berry phase associated with an electron having even number of flux quanta attached to it can be removed to the dynamical phase and the residual Berry phase of the QE is computed considering the reduced magnetid field. Indeed this helps to improve the result obtained by Kjønsberg and Myrheim [1] at $\nu=1 / 3$.

In sec.II we shall briefly sketch the model of QHE on the basis of Berry phase approach 5, 6] incorporating the fact that even number flux quanta can be gauged away when the phase is removed to the dynamical phase. In sec.III we shall analyze Laughlin wave functions for $\mathrm{QE}$ and $\mathrm{QH}$ incorporating this aspect of Berry phase. In sec.IV we shall reproduce the numerical result of Kjønsberg and Myrheim [1]

\footnotetext{
*Electronic address: banasri@isical.ac.in

${ }^{\dagger}$ Electronic address: pratul@isical.ac.in

${ }^{\ddagger}$ Electronic address: sarmi $30 @$ rediffmail.com
} 
at $\nu=1 / 3$ taking into account this modification.

\section{FRACTIONAL QUANTUM HALL EFFECT AND BERRY PHASE}

In some earlier papers [5, 6] we have analyzed the sequence of quantum Hall states from the viewpoint of chiral anomaly and Berry phase. In our approach, we have considered the spherical geometry which was first used by Haldane [7] where the electrons are confined on the surface of a sphere of large radius $R$ with a magnetic monopole of strength $\mu$ at the centre. In this geometry, the single electron is represented as a spin $S$, the orientation of which indicates the point on the sphere about which the state is localized. The angular momentum shells of the spherical geometry are the analogy of the Landau levels (LL) of the planar geometry. The angular momentum relation here is given by

$$
\mathbf{J}=\mathbf{r} \times \mathbf{p}-\mu \hat{\mathbf{r}}, \mu=0, \pm 1 / 2, \pm 1, \pm 3 / 2 \cdots .
$$

The lowest Landau level (LLL) is given by the $L=|\mu|$ shell.

It may be remarked here that as $|\mu|$ here corresponds to the monopole strength we can relate this with the Berry phase. Indeed $\mu=1 / 2$ corresponds to one flux quantum and the flux through the sphere when there is a monopole of strength $\mu$ at the centre is $2 \mu$. The Berry phase of a fermion of charge $q$ when it moves in a closed path is given by $e^{i \phi_{B}}$ with $\phi_{B}=2 \pi q N$, here $N$ is the number of flux quanta enclosed by the loop traversed by the particle.

If $\mu$ is an integer, we can have a relation of the form

$$
\mathbf{J}=\mathbf{r} \times \mathbf{p}-\mu \hat{\mathbf{r}}=\mathbf{r}^{\prime} \times \mathbf{p}^{\prime}
$$

which indicates that the Berry phase associated with integer $\mu$ may be unitarily removed to the dynamical phase. This suggests that the attachment of $2 p$ vortices(magnetic flux lines) with $p$ an integer to an electron effectively leads to the removal of Berry phase to the dynamical phase. So, FQH states with $2 \mu_{e f f}=2 p+1$ can be viewed as if one vortex is attached to the electron. From the Dirac quantization condition $e \mu_{e f f}=\frac{1}{2}$, we can identify the filling factor $\nu$ with $\frac{1}{2 \mu_{e f f}}$ corresponding to the charge of the particle given by $\nu e$. For a higher Landau level we can consider the Dirac quantization condition $e \mu_{\text {eff }}=\frac{1}{2} n$, with $n$ being a vortex of strength $2 \ell+1$. This can generate FQH states having the filling factor of the form $\frac{n}{2 \mu_{e f f}}$ where both $n$ and $2 \mu_{\text {eff }}$ are odd integers. Indeed, we can write the filling factor as [, [6]

$$
\nu=\frac{n}{2 \mu_{e f f}}=\frac{1}{\frac{2 \mu_{e f f} \mp 1}{n} \pm \frac{1}{n}}=\frac{n}{2 p n \pm 1}
$$

where $2 \mu_{e f f} \mp 1$ is an even integer given by $2 p n$. In this scheme, the FQH states with $\nu$ having the form

$$
\nu=\frac{n^{\prime}}{2 p n^{\prime} \pm 1}
$$

where $n^{\prime}$ an even integer can be generated through particle-hole conjugate states

$$
\nu=1-\frac{n}{2 p n \pm 1}=\frac{n(2 p-1) \pm 1}{2 p n \pm 1}=\frac{n^{\prime}}{2 p n^{\prime} \pm 1}
$$

These FQH states having even numerator and odd denominator filling factors can be considered as particle-hole conjugate states in this scheme.

The segregation of even number of vortices helps us to consider the removal of the corresponding phase factor to the dynamical phase. To see this explicitly, we take resort to the planar geometry when the ground state wave function is given by Laughlin wave function. The ground state wave function for an $N$-particle system at the filling factor $\nu=\frac{1}{m}=\frac{1}{2 p+1}$ is given by

$$
\psi_{m}=\prod_{i<j}\left(z_{i}^{*}-z_{j}^{*}\right)^{m} e^{-\frac{1}{2} \sum\left|z_{i}\right|^{2}}
$$


where $z=\frac{x+i y}{\sqrt{2} l}, l$ being the magnetic length $l=\frac{1}{\sqrt{e B}}(\hbar=c=1)$. Now segregating the even number of vortices we consider the phase of the Jastrow factor $\prod_{i<j}\left(z_{i}^{*}-z_{j}^{*}\right)^{2 p}$. We can display the phases due to the Jastrow factor by writing

$$
\prod_{i<k}\left(z_{i}^{*}-z_{k}^{*}\right)^{2 p}=e^{i 2 p \sum_{j<k} \phi_{j k}} \prod_{j<k}\left|z_{j}-z_{k}\right|^{2 p}
$$

where

$$
\phi_{j k}=i \ln \frac{z_{j}^{*}-z_{k}^{*}}{\left|z_{j}-z_{k}\right|}
$$

This effectively leads to the many body wave function of the electrons $\psi_{e}$ to the transformed wave function through the relation

$$
\phi_{t r}\left\{\vec{r}_{i}\right\}=\phi_{e}\left\{\vec{r}_{i}\right\} \prod_{i<j}\left(\frac{z_{j}^{*}-z_{k}^{*}}{\left|z_{j}-z_{k}\right|}\right)^{2 p}
$$

The unitary transformation (9) of the wave function, which may be described as a singular gauge transformation requires a corresponding transformation of the Hamiltonian. The Schrödinger equation can now be written as $[8]$

$$
\begin{gathered}
{\left[\frac{1}{2 m_{b}} \sum_{i}\left(p_{i}+e \mathbf{A}\left(\mathbf{r}_{i}\right)-e \mathbf{a}\left(\mathbf{r}_{i}\right)\right)^{2}+V\right] \prod_{j<k}\left|z_{j}-z_{k}\right|^{2 p}\left(z_{i}^{*}-z_{j}^{*}\right) e^{-\frac{1}{2} \sum\left|z_{i}\right|^{2}}} \\
=E \prod_{j<k}\left|z_{j}-z_{k}\right|^{2 p}\left(z_{i}^{*}-z_{j}^{*}\right) e^{-\frac{1}{2} \sum\left|z_{i}\right|^{2}}
\end{gathered}
$$

where $m_{b}$ is the electron band mass. It is noted that here we have introduced the additional vector potential $\mathbf{a}\left(\mathbf{r}_{i}\right)$ that simulates the effect of the phases of the Jastrow factor. In fact we have

$$
\mathbf{a}\left(\mathbf{r}_{i}\right)=\frac{2 p}{2 \pi} \phi_{o} \sum_{i}^{\prime} \vec{\nabla}_{i} \phi_{i j}
$$

where the prime denotes the condition $i \neq j$. Here $\phi_{0}$ is the unit magnetic flux quanta given by $\phi_{0}=\frac{h c}{e}$. The corresponding magnetic field is

$$
\vec{b}_{i}=2 p \phi_{0} \hat{z} \sum_{i}^{\prime} \delta^{2}\left(\mathbf{r}_{i}-\mathbf{r}_{j}\right)
$$

Thus the phase of the Jastrow factor is equivalent to each electron seeing a flux tube of $2 p \phi_{0}$ on other electron. In a translationally invariant system, the mean field Hamiltonian $H_{0}$ may be obtained by replacing $\vec{b}(\mathbf{r})$ by the mean value

$$
<\vec{b}>=2 \pi 2 p n_{e}
$$

where $n_{e}$ is the average electron density and by ignoring the potential energy $V$, we may now write

$$
H_{0}=\frac{1}{2 m_{b}} \int \psi^{\dagger}(\mathbf{r})\left[-i \nabla+A^{*}(\mathbf{r})\right]^{2} \psi(\mathbf{r}) d^{2} r
$$

where $\nabla \times A^{*}=B^{*}=B-2 \pi 2 p n_{e}$.

Thus the effect of the removal of the Berry phase associated with even number of flux quanta to the dynamical phase is transcribed by the reduction of the magnetic field $B$ to $B^{*}$ with

$$
\left|B^{*}\right|=\frac{B}{2 p n+1}
$$


The Berry phase acquired by an electron when it traverses a closed path encircling an area $A$ in relation to the reduced field $B^{*}$ is given by

$$
\phi^{*}=2 \pi \frac{B^{*} A}{\phi_{0}}
$$

For a quantum Hall state with filling factor $\nu=\frac{n}{2 m n+1}=\frac{1}{2 m+1 / n}$, it appears that when even number of flux quanta is gauged away, the electron is attached with a magnetic flux quantum having strength $1 / n$ and so we will have the phase in the reduced field $B^{*}$

$$
\phi^{*}=2 \pi \frac{n B^{*} A}{\phi_{0}}
$$

In the field $B$, this gives rise to

$$
\begin{aligned}
\phi & =2 \pi \frac{n B A}{(2 p n+1) \phi_{0}} \\
& =2 \pi \frac{n}{(2 p n+1)} N
\end{aligned}
$$

where $N$ is the number of flux quanta enclosed by the path. So when a particle having the total number of flux quanta $|2 p+1 / n|$ in the field $B$ traverses a closed path encircling $N$ number of such particles, the Berry phase is given by

$$
\phi=2 \pi \frac{n}{(2 p n+1)} N
$$

It is noted that in our classification scheme we have identified the filling factor $\nu$ with the local charge of the particle $q=\nu$ (in unit of e) and so we can define the Berry phase by

$$
\phi=2 \pi q N
$$

Evidently this is analogous to the Berry phase acquired by an electron when it traverses a closed path encircling $N$ number of electrons but is different from that of a composite fermion in the CF picture. From eqn.(19) it is observed that when a particle attached with $2 p+\frac{1}{n}$ flux quanta moves about another one traversing half circle the phase is $\phi=\pi \frac{n}{2 p n+1}$ so that the statistics parameter $\theta=\frac{n}{2 p n+1}$.

\section{CHARGE AND STATISTICS OF THE QUASIELECTRONS AND QUASIHOLES}

The Laughlin wave function is characterized by the fact that for one quasihole $(\mathrm{QH})$ at the position $z_{0}$ it is given by

$$
\psi_{z_{0}}^{1 q h}=\psi_{0} \triangle^{* m} \prod_{i=1}^{N}\left(z_{i}^{*}-z_{0}^{*}\right)
$$

where

$$
\begin{aligned}
& \psi_{0}=e^{-\frac{1}{2} \sum_{i=1}^{N}\left|z_{i}\right|^{2}} \\
& \triangle=\prod_{j<k}\left(z_{j}-z_{k}\right)
\end{aligned}
$$

and $m=1 / \nu, \nu$ being the filling factor. It is noted that $z$ is here given by $\frac{x+i y}{\sqrt{2} l}, l$ being the magnetic length. For a quasielectron $(\mathrm{QE})$ at $z_{0}$ the corresponding wave function is given by

$$
\psi_{z_{0}}^{1 q e}=\psi_{0} \prod_{i=1}^{N}\left(\partial_{z_{i}^{*}}-z_{0}\right) \triangle^{* m}
$$


To study the statistics parameter we need to compute the phase related to the motion of one $\mathrm{QE}(\mathrm{QH})$ around another $\mathrm{QE}(\mathrm{QH})$. So, we need the construction of two QHs and QEs wave functions. For two QHs at the positions $z_{a}$ and $z_{b}$, the wave function is

$$
\psi_{z_{a}, z_{b}}^{2 q h}=\psi_{0} \triangle^{* m} \prod_{i=1}^{N}\left(z_{i}^{*}-z_{a}^{*}\right)\left(z_{i}^{*}-z_{b}^{*}\right)
$$

Similarly for the QEs at $z_{a}$ and $z_{b}$, the wave function is given by

$$
\psi_{z_{a}, z_{b}}^{2 q e}=\psi_{0}\left(\prod_{i=1}^{N}\left(\partial_{z_{i}^{*}}-z_{a}\right)\left(\partial_{z_{i}^{*}}-z_{b}\right)\right) \triangle^{* m}
$$

Now following Kjønsberg and Myrheim [1] we consider a quasiparticle excitation (QH or QE) of charge $q$ localized at the position $z_{0}$ and is described by the normalized wave function $\left\langle z_{1}, \ldots \ldots z_{N} \mid z_{0}\right\rangle$. If this quasiparticle (QP) is moved around a closed path there arises the Berry phase. If the path is a circle around the origin parameterized by $z_{0}=r e^{i \phi}$ with $\phi$ running from 0 to $2 \pi$, the Berry connection is then defined by

$$
\frac{d \beta_{1}}{d \phi}=i<z_{0}\left|\partial_{\phi}\right| z_{0}>
$$

The charge $q$ of the quasiparticle is determined by setting the Berry phase equal to the AharonovBohm phase corresponding to the same path. Evidently in a finite system the charge defined in this way will depend on the distance $r$ from the origin but it gives a reasonable value when the circles are well within the droplet. If the quasiparticle is described by a normalized $N$ - particle state of the form

$$
\left|z_{0}>=\frac{1}{\sqrt{I_{1}}} \sum_{l=0}^{\alpha} z_{0}^{l} a_{l}\right| l>
$$

where $a_{l}$ are expansion coefficients, $\mid l>$ are orthonormal basis states and $I_{1}$ is the normalization factor

$$
I_{1}=\sum_{l=0}^{\alpha} r^{2 l}\left|a_{l}\right|^{2}
$$

The expression for charge is given by $[1]$

$$
\frac{q}{e}=\frac{1}{r^{2}} \frac{d \beta_{1}}{d \phi}=-\frac{d}{d r^{2}} \ln I_{1}
$$

When there are two quasiparticle excitations simultaneously located symmetrically about the origin at the positions $\pm z_{0}$ with the parametrization $z_{0}=r e^{i \phi}$ we can let $\phi$ run from 0 to $\pi$ which describes the interchange of two quasiparticles. If the quasiparticle state is described by a state analogous to (26)

$$
\left|z_{0},-z_{0}>=\frac{1}{\sqrt{I_{2}}} \sum_{l=0}^{\alpha} z_{0}^{l} b_{l}\right| l>
$$

then a Berry phase connection $\frac{d \beta_{2}}{d \phi}$ corresponding this interchange is given by

$$
\frac{d \beta_{2}}{d \phi}=i<z_{0},-z_{0}\left|\partial_{\phi}\right| z_{0},-z_{0}>
$$

We can now define the statistics parameter by subtracting the single particle contribution due to the magnetic field

$$
\begin{aligned}
-\theta & =\frac{1}{\pi}\left(\beta_{2}(\pi)-2 \beta_{1}(\pi)\right) \\
& =\frac{d}{d \phi}\left(\beta_{2}-2 \beta_{1}\right) \\
& =-r^{2} \frac{d}{d r^{2}}\left(\ln I_{2}-2 \ln I_{1}\right)
\end{aligned}
$$


Specifying $I$ for quasielectron (quasihole) by $I^{1 q h}, I^{2 q h}, I^{1 q e}, I^{2 q e}$, we can write the following expression for charge and statistics parameter [1]

$$
\begin{aligned}
\frac{q^{q h}}{e} & =\frac{d}{d r^{2}} \ln I^{1 q h} \\
\frac{q^{q e}}{e} & =-\frac{d}{d r^{2}} \ln I^{1 q e} \\
\theta^{q h} & =-r^{2} \frac{d}{d r^{2}}\left(\ln I^{2 q h}-2 \ln I^{1 q h}\right) \\
\theta^{q e} & =r^{2} \frac{d}{d r^{2}}\left(\ln I^{2 q e}-2 \ln I^{1 q e}\right)
\end{aligned}
$$

Now from the wavefunctions for $\psi_{z_{0}}^{1 q h}, \psi_{z_{0}}^{1 q e}, \psi_{z_{a}, z_{b}}^{2 q h}, \psi_{z_{a}, z_{b}}^{2 q e}$ given by eqns.(21), (22), (23) and (24), we have [1]

$$
\begin{aligned}
I^{1 q h}\left(r^{2}\right) & =\int d^{2 N} z \psi_{0}^{2}|\triangle|^{2 m} \prod_{k=1}^{N}\left|z_{k}-z_{0}\right|^{2} \\
I^{1 q e}\left(r^{2}\right) & =\int d^{2 N} z \psi_{0}^{2}|\triangle|^{2 m} \prod_{k=1}^{N}\left(\left|z_{k}-z_{0}\right|^{2}-1\right) \\
I_{z_{a}, z_{b}}^{2 q h} & =\int d^{2 N} z \psi_{0}^{2}|\triangle|^{2 m} \prod_{k=1}^{N}\left|\left(z_{k}-z_{a}\right)\left(z_{k}-z_{b}\right)\right|^{2} \\
I_{z_{a}, z_{b}}^{2 q e} & =\int d^{2 N} z \psi_{0}^{2}|\triangle|^{2 m} \prod_{k=1}^{N}\left(\left|z_{k}-z_{a}\right|^{2}\left|z_{k}-z_{b}\right|^{2}-\left|2 z_{k}-z_{a}-z_{b}\right|^{2}+2\right)
\end{aligned}
$$

From the above results obtained by Kjønsberg and Myrheim [1], we now consider the case when the quasiparticle is subjected to the reduced magnetic field $B^{*}$ after the removal of the Berry phase associated with even number of flux quanta to the dynamical phase. It is noted that when the magnetic field $B$ is reduced to $B^{*}$, the corresponding magnetic length is changed from $l$ to $l^{*}=l \sqrt{m}$ where $m$ is given by $2 p n+1$ corresponding to the filling factor $\nu=\frac{n}{2 p n+1}$. Now considering that the ground state can be thought as the "vacuum", we put the quasiparticle at the position $z_{0}$ such that it is subjected to the reduced magnetic field $B^{*}$. The corresponding change in the value of the magnetic length $l^{*}=l \sqrt{m}$, at filling factor $\nu=\frac{1}{m}=\frac{1}{2 p+1}$ will modify the expressions $I_{1}^{q h}$ and $I_{1}^{q e}$ given by eqns.(36) and (37)

$$
\begin{aligned}
I_{1}^{q h} & =\int d^{2 N} z \psi_{0}^{2}|\triangle|^{2 m} \prod_{k=1}^{N} m\left|z_{k}-z_{0}\right|^{2} \\
I_{1}^{q e} & =\int d^{2 N} z \psi_{0}^{2}|\triangle|^{2 m} \prod_{k=1}^{N}\left(m\left|z_{k}-z_{0}\right|^{2}-1\right)
\end{aligned}
$$

Similarly for this quasiparticles, the expressions for $I_{2}^{q h}$ and $I_{2}^{q e}$ will be modified as

$$
\begin{aligned}
I_{2}^{q h} & =\int d^{2 N} z \psi_{0}^{2}|\triangle|^{2 m} \prod_{k=1}^{N} m^{2}\left|\left(z_{k}-z_{a}\right)\left(z_{k}-z_{b}\right)\right|^{2} \\
I_{2}^{q e} & =\int d^{2 N} z \psi_{0}^{2}|\triangle|^{2 m} \prod_{k=1}^{N} m^{2}\left(\left|z_{k}-z_{a}\right|^{2}\left|z_{k}-z_{b}\right|^{2}-m\left|2 z_{k}-z_{a}-z_{b}\right|^{2}+2\right)
\end{aligned}
$$

These changes in the expressions due to the change in the normalization factor for $z$ will modify the numerical results obtained by Kjønsberg and Myrheim [1]. Indeed, the crux of the problem related to the differences in numerical value for the charge and statistics parameter for quasihole and quasiparticles 
in ref.[1] lies in the expressions for $I_{1}^{q h}\left(I_{2}^{q h}\right)$ and $I_{1}^{q e}\left(I_{2}^{q e}\right)$. In $I_{1}^{q e}$ we have the extra factor $(-1)$ associated with the term $\left|z_{k}-z_{0}\right|^{2}$. If we could neglect this factor $(-1)$ then $I_{1}^{q h}$ and $\left(I_{1}^{q e}\right)$ would have been identical. However since the average number of electrons within unit distance from an arbitrary point $z_{0}$ assuming constant density within the electron droplet is $\pi / m$, it is very close to 1 for $m=3$. But when we consider the effect of the reduced magnetic field $B^{*}$ on the quasiparticle, this average number of electrons will change to $n \pi$ for filling factor $\frac{n}{2 p n+1}$ which is expected to improve the situation.

\section{NUMERICAL RESULTS}

From our above analysis it appears that when the quasiparticle (QH or $\mathrm{QE})$ is subjected to the reduced magnetic field $B^{*}$, the expression for $z_{0}=r e^{i \phi}$ in unit of $l^{*}=l \sqrt{m}$ will change the parameter $r$ to $\tilde{r}=r / \sqrt{m}$. Similarly, the parameter related to the distance between two quasiparticles will also be modified. Taking into account this modification we have reproduced here the numerical results for the charge $q / e$ and statistics parameter $\theta$ obtained by Kjønsberg and Myrheim [1] in Figs. 1,2,3 and 4. It is to be remarked that as in the present formalism a quasiparticle is not a conventional point like anyon, rather it has an extended structure as certain magnetic flux lines are attached to it, the size of a quasiparticle can be taken to be of the order of one magnetic length. In view of this we plot here the values from $\tilde{r}=1$.

It is found that, as expected the charge and statistics parameter fits very well with the values $e / 3$ and $1 / 3$ respectively in case of quasiholes as shown in figs 1 and 3 . It may be noted that below $\tilde{r}<1$, we have observed appreciable deviation from the value $q=e / 3$ and $\theta=1 / 3$ as has been shown in fig 2 and $6(r=\tilde{r} \sqrt{m})$ reported in ref.[1].

However, in case of quasielectrons, we note that the slight deviation from the exact value for the charge $q=e / 3$ is minimal for different values of $N$ in the range $\tilde{r}=1$ to 3 . Similarly the average value of the statistics parameter $\theta$ for quasielectrons becomes close to the value $1 / 3$ within the range $\tilde{r}=1$ to 3 and it deviates largely when $\tilde{r}$ increases. In fact it is expected that we should consider the result within this range as the edge effect becomes prominent beyond this. In the perspective of spherical geometry the size of the droplet $R$ is given by $R \sim l \sqrt{N}$. To be consistent with the distance coordinates $\tilde{r}=r / \sqrt{m}$, we have to consider the droplet size $\tilde{R} \sim \frac{l}{\sqrt{m}} \sqrt{N}$. So even for $N=100$, with $m=3$, we note that $\tilde{R}$ is of the order of 5 units of magnetic length. So it is expected that the edge effect will be minimal only in the range $\tilde{r}=1$ to 3 . Indeed it has been observed that all important physics at the edge is not one dimensional but leads to the formation of striped phase at $\nu=1 / 3$ caused by long ranged Coulomb interaction [9]. The amplitude of the charge density oscillations decays slowly only as a power law with the distance from the edge. It is found that at $\nu=1 / 3$ the quantum Hall edge undergoes a reconstruction as the background potential softens, whereas quantum Hall edges at higher filling factors are robust against reconstruction [10]. Since the edge physics at $\nu=1 / 3$ is not confined to the boundary but extends to the bulk, we should consider our results within a specific range [11]. However, as the size of the droplet varies as $R \sim \sqrt{N}$, it is expected that the situation will improve for large $N$.

In view of these considerations we can argue that the deviation from the exact value of $e / 3$ and $1 / 3$ for charge and statistics parameter at $\nu=1 / 3$ is to be interpreted in terms of the nature of the quasiparticles and edge physics and it is expected that we will have exact value in the thermodynamic limit. 


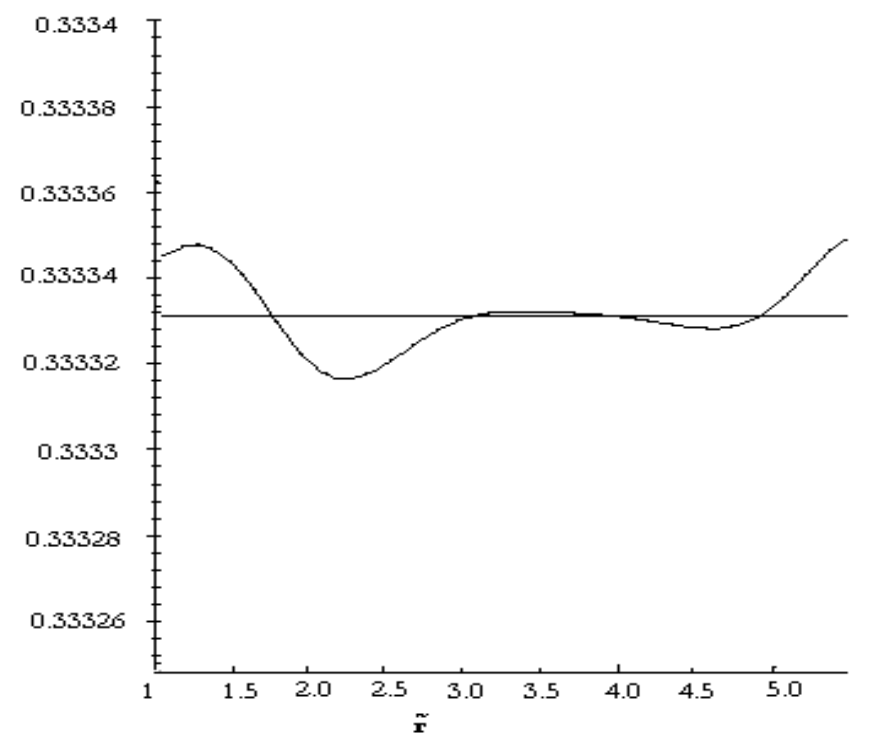

FIG. 1: The quasihole charge, $q^{q h} / e$, compared to $1 / 3$, for 100 electrons. 


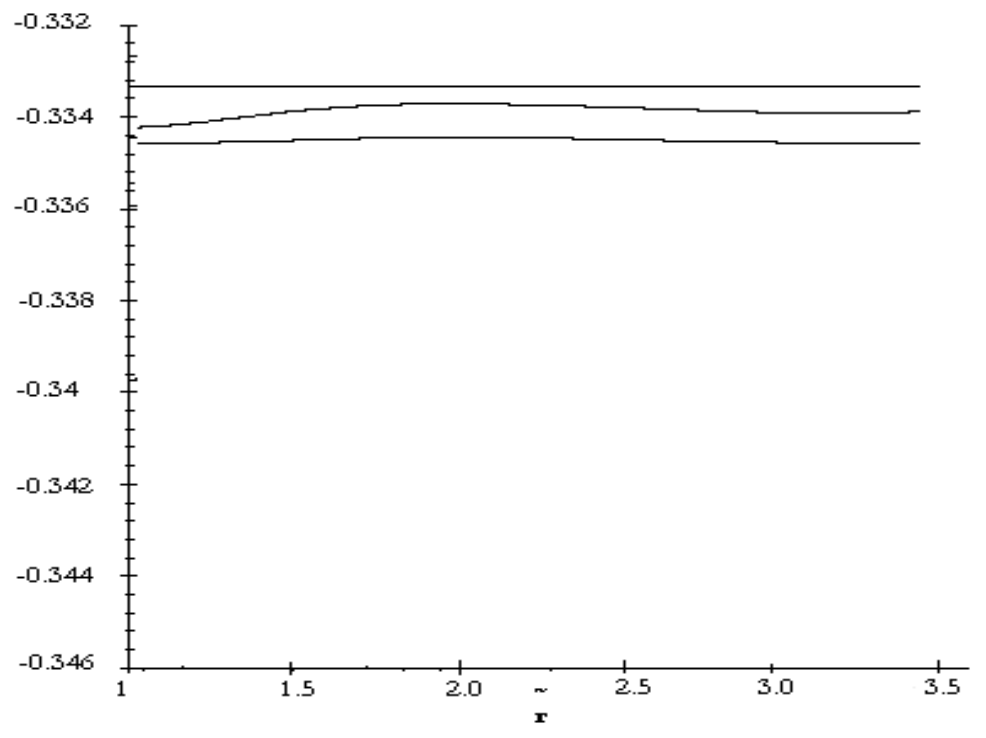

FIG. 2: Quasielectron charge, $q^{q e} / e$, for 100 and 200 electrons. The highest curve is the constant $-1 / 3$ 


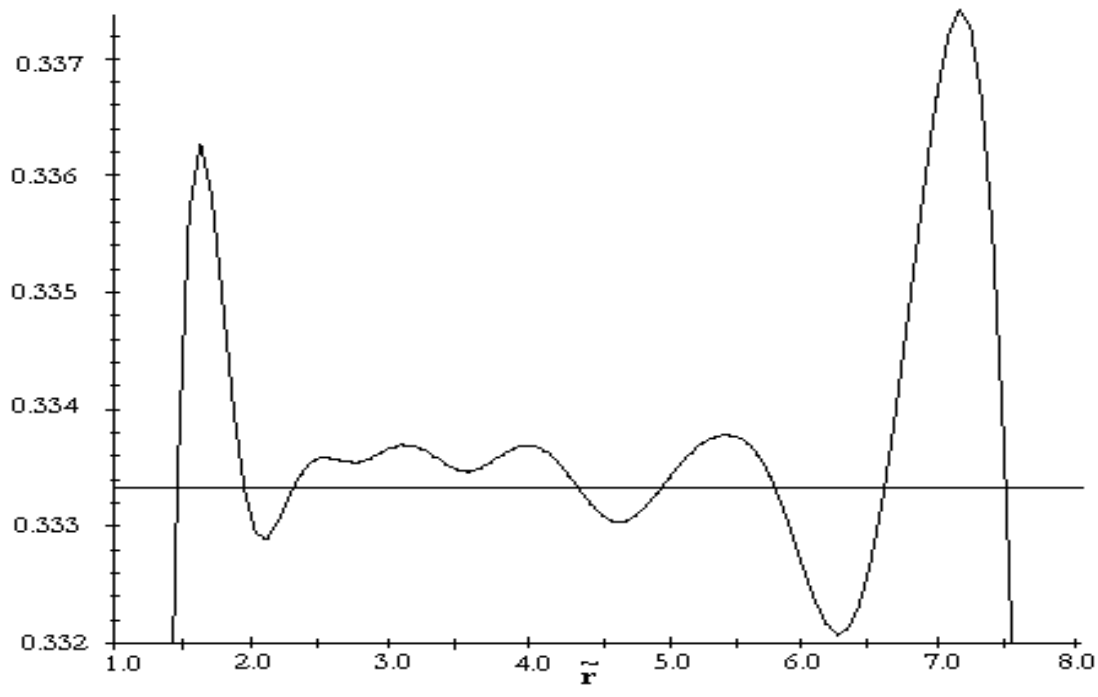

FIG. 3: Quasihole statistics parameter $\nu^{q h}$ for 100 electrons, compared to $1 / 3$, emphasizing the bulk behavior. 


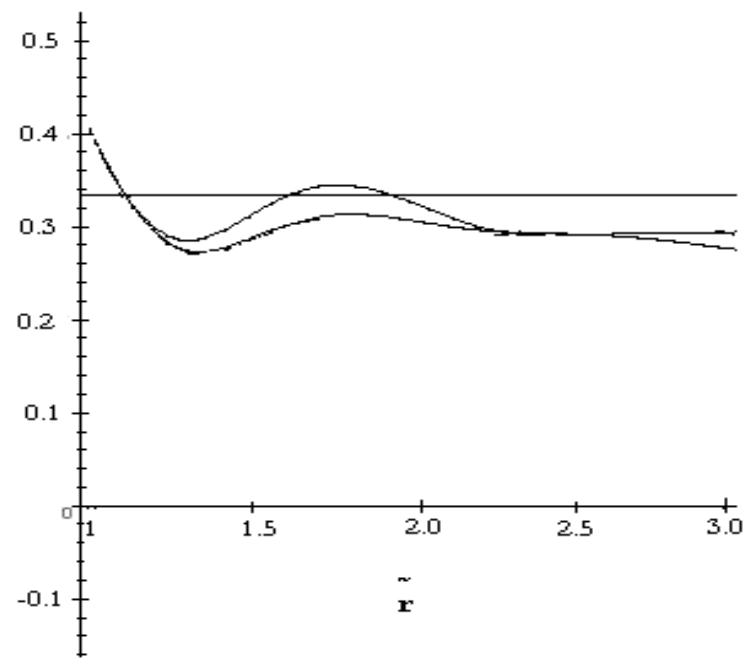

FIG. 4: Quasielectron statistics parameter $\nu^{q e}$ for 100 and 200 electrons. The 200 electron curve overshoots the horizontal line $1 / 3$ at $\tilde{r} \approx 1.732$. 


\section{DISCUSSION}

We have pointed out that when the effect of the removal of the Berry phase associated with even number of flux quanta to the dynamical phase is taken into account, we get better estimates of the charge and statistics parameter of the quasiparticles. Again taking into account the edge effect in the bulk which is found to be very prominent at the filling factor $\nu=1 / 3$, the departure from the expected value for the charge and statistics parameter for quasielectrons $q=e / 3$ and $\theta=1 / 3$ can be explained. It may be recalled that in the composite fermion picture the value of the statistics parameter $\theta$ is predicted to be $2 / 3$ for $\nu=1 / 3$. Though the numerical computation agrees with the magnitude, it differs in sign. This raises doubt on the fundamental interpretation of the CF physics. To cope with this problem it has been suggested that the insertion of a certain CFQP at a point perturbs the other CFQP by pushing it to slightly outward from the loop.

It is to be remarked that the present formalism does not incorporate the conventional point anyon picture. Indeed quasiparticles are particles attached with magnetic flux quanta and hence should be treated as extended bodies. Fractional charge and hence fractional statistics is found to be an outcome of Dirac quantization condition. It may be noted that in this formalism the very definition of filling factor $\nu$ is associated with the charge $\nu e$ derived from the Dirac quantization relation. This is consistent with experimental results and is identical with the predictions of the CF theory in case of FQH states with $\nu=1 / m$ ( $m$ being an odd integer). But there is a controversy regarding the charge of quasiparticles in the FQH states with the filling factor $\nu=n / m$. In the CF model the predicted charge of the quasiparticles with $\nu=n / m$ is always $1 / m$ which is supposed to be supported by experiments at a bit higher temperature [12] but is in contrast to the experimental result [13] where it is shown that the charge of the quasiparticles are $e / 3,2 e / 5$ and $3 e / 7$ at $\nu=1 / 3,2 / 5$ and $3 / 7$ at extremely low temperature. In this context we may add that the Dirac quantization condition which is a consequence of quantum field theory at $T=0$ (no finite temperature effect is taken into account) our result is expected to be valid in the close vicinity of $T=0$. However at higher temperature it may happen that for quasiparticles with $\nu=n / m$, the system is dissociated into $n$ copies of quasiparticles with charge $e^{*}=e / m$ [14].

Finally we may add that as it has been discussed in the previous section, the edge effect is most prominent at $\nu=1 / 3$, it is expected that the numerical experiments will give better result for other filling factors such as $2 / 5,3 / 5,3 / 7$ and so on. In a future study we shall take up this issue.

[1] H. Kjønsberg and J. Myrheim, Int. J. Mod. Phys. A 34, 537 (1999).

[2] H. Kjønsberg and M. Leinaas, Nucl. Phy. B 559705 (1999).

[3] G. S. Jeon, K. L. Graham and J. K. Jain, Phys. Rev. Lett. 91, 036801 (2003).

[4] G. S. Jeon, K. L. Graham and J. K. Jain, cond-mat/0407137 (2004)

[5] B. Basu and P. Bandyopadhyay, Int. J. Mod. Phys. B 122649 (1998).

[6] B. Basu and P. Bandyopadhyay, Int. J. Mod. Phys. B 1249 (1990).

[7] F. D. M. Haldane, Phys. Rev. Lett. 51, 605 (1983).

[8] B. I. Halperin, Perspective in Quantum Hall Effect (Ed.s S. Das Sarma, A Pinczuk, Jojn Wiley and Sons, Inc.,N. Y. p-225. (1997))

[9] F. V. Tsiper and V. J. Goldman, Phys. Rev. B 64, 165311 (2001).

[10] Y. N. Jogelker, H. K. Nguyen and G. Murthy, cond-mat/0303359 (2003)

[11] B. Basu and P. Bandyopadhyay, Fractional Quantum Hall Edge Electrons, Chiral Anomaly and Berry Phase; preprint : cond-mat/0401418

[12] M. Reznikov, R. de Picciotto, T. G. Griffiths, M Heiblem and V. Umansky, Nature 399, 238 (1999).

[13] Y. C Chung, M Heiblem and V. Umansky, Phys. Rev. Lett. 91, 216804 (2003).

[14] B. Basu and P. Bandyopadhyay, Phys. Lett. A 337, 81 (2005). 